TITLE:

\title{
Near-field simulation of obliquely deposited surface-enhanced Raman scattering substrates
}

\section{$\operatorname{AUTHOR}(\mathrm{S}):$}

Jen, Yi-Jun; Suzuki, Motofumi; Wang, Yung-Hsiang; Lin, Meng-Jie

\section{CITATION:}

Jen, Yi-Jun ...[et al]. Near-field simulation of obliquely deposited surface-enhanced Raman scattering substrates. Journal of Applied Physics 2012, 112(11): 113111.

\section{ISSUE DATE:}

2012-12-12

URL:

http://hdl.handle.net/2433/187970

\section{RIGHT:}

(c) 2012 American Institute of Physics. This article may be downloaded for personal use only. Any other use requires prior permission of the author and the American Institute of Physics. 


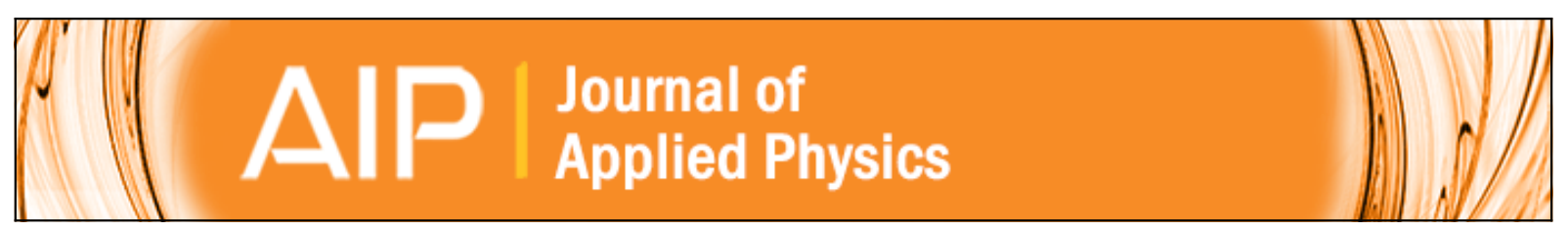

\section{Near-field simulation of obliquely deposited surface-enhanced Raman scattering substrates}

Yi-Jun Jen, Motofumi Suzuki, Yung-Hsiang Wang, and Meng-Jie Lin

Citation: Journal of Applied Physics 112, 113111 (2012); doi: 10.1063/1.4769806

View online: http://dx.doi.org/10.1063/1.4769806

View Table of Contents: http://scitation.aip.org/content/aip/journal/jap/112/11?ver=pdfcov

Published by the AIP Publishing

\section{Articles you may be interested in}

Gold on graphene as a substrate for surface enhanced Raman scattering study

Appl. Phys. Lett. 97, 163111 (2010); 10.1063/1.3505335

Tuning plasmonic interaction between gold nanorings and a gold film for surface enhanced Raman scattering Appl. Phys. Lett. 97, 163106 (2010); 10.1063/1.3504187

Ultradense gold nanostructures fabricated using hydrogen silsesquioxane resist and applications for surfaceenhanced Raman spectroscopy

J. Vac. Sci. Technol. B 27, 2640 (2009); 10.1116/1.3253610

Revisiting the separation dependent surface enhanced Raman scattering

Appl. Phys. Lett. 93, 173106 (2008); 10.1063/1.3009301

Surface enhanced Raman scattering in the near infrared using metal nanoshell substrates

J. Chem. Phys. 111, 4729 (1999); 10.1063/1.479235

\section{AlP Re-register for Table of Content Alerts}




\title{
Near-field simulation of obliquely deposited surface-enhanced Raman scattering substrates
}

\author{
Yi-Jun Jen, ${ }^{1, a)}$ Motofumi Suzuki, ${ }^{2, b)}$ Yung-Hsiang Wang, ${ }^{1}$ and Meng-Jie Lin ${ }^{1}$ \\ ${ }^{1}$ Department of Electro-Optical Engineering, National Taipei University of Technology, Taipei 106, Taiwan \\ ${ }^{2}$ Department of Micro Engineering, Kyoto University, Kyoto 606-8501, Japan
}

(Received 30 August 2012; accepted 17 November 2012; published online 12 December 2012)

\begin{abstract}
A semicontinuous gold layer on a template with a zig-zag structure has been demonstrated to be a highly sensitive substrate for surface-enhanced Raman scattering (SERS). Obliquely deposited gold layers on different templates yield different SERS strengths. In this work, a finite-difference time-domain method is utilized to analyze the electric field distribution within the gold layer when the film is illuminated by light. The random gold nanostructures are scanned and transformed into binary images to allow them to be simulated. The field intensities and SERS enhancement factors of different samples are calculated and compared. Our analysis results reveal the near-field characteristics of strong SERS and agree closely with experimental measurements. (C) 2012 American Institute of Physics. [http://dx.doi.org/10.1063/1.4769806]
\end{abstract}

\section{INTRODUCTION}

Surface-enhanced Raman scattering (SERS) is a sensitive spectroscopic technique that provides a spectral fingerprint of the adsorbed analyte in a nondestructive way. SERS has been applied in several important fields including the detection of chemical and biological agents, ${ }^{1,2}$ biomedical diagnostics, ${ }^{3,4}$ DNA analysis, ${ }^{5,6}$ and $\mathrm{pH}$ sensing. ${ }^{7}$ Compared to normal Raman scattering, SERS exhibits a much greater sensitivity. SERS has the advantage that it can sense lowconcentration chemical species. On the other hand, SERS is also able to resolve multiple analytes simultaneously.

Local field enhancement in the vicinity of nanostructured noble metal surfaces is mediated by localized plasmons in the metals under illumination and is essential for various surface enhancement effects such as surface enhanced Raman scattering (SERS) and fluorescence (SEF). Thus, for improvement of SERS and SEF, it is important to find nanostructures with optimum sizes, shapes, and arrangements to efficiently enhance local electric fields. Recently, it has become possible to manufacture monodispersed colloidal nanoparticles with well-controlled sizes and shapes through chemical processes. ${ }^{8}$ Advanced lithographic techniques enable the fabrication of periodic nanoparticle arrays with excellent SERS properties. ${ }^{9,10}$ In the cases of clusters of a small number of nanoparticles or periodic arrays of nanoparticles, the contribution of the localized plasmons and of the enhanced local field to the optical and SERS properties are well understood theoretically.

On the other hand, from a practical point of view, random nanostructures, such as aggregates of a large number of colloidal nanoparticles or high-density arrays of granular nanoparticles with irregular shapes, are also useful for SERS. ${ }^{11-14}$ The properties of these random nanostructures are typically discussed qualitatively based on an analogy

\footnotetext{
${ }^{a)}$ Electronic mail: jyjun@ntut.edu.tw.

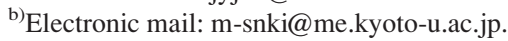

with existing theoretical results for simple nanostructures with regular shapes. The influence of irregularities in sizes, shapes, and arrangement on the local field enhancement has not been well understood. In particular, the interaction between nanoparticles distributed with random spacing has been hardly considered at all. A quantitative understanding of the influence of the randomness on the plasmonic and local field properties will lead us to optimized structures for applications and to novel phenomena that are specific to random nanostructures.

Much of the recent work on simulating complex metaldielectric structures has been done to increase understanding of the details of local surface plasmon resonances according to the known geometry. For a periodic nanostructure, the near-field simulation can be performed with the finitedifference time-domain (FDTD) method. The hot spots, which are the locations associated with electromagnetic field enhancement, are indicated and regarded as the main source for SERS. However, analyzing irregular nanostructures is rarely done, but in this case, it is necessary. In order to understand the local field enhancement near random nanostructures, we must establish theoretical methods to treat realistic random nanostructures, and we require systematic experiments that can be compared with the theoretical treatments. Fortunately, granular nanoparticle arrays prepared by oblique angle deposition technique show excellent and systematic SERS properties,${ }^{14,15}$ which are a good index of local field enhancement, which is dependent on their preparation conditions.

In this work, we apply the FDTD method to interpret SERS signals from four different semicontinuous gold films. SERS signals from the four different semicontinuous gold films have been measured and reported. ${ }^{15}$ The plasmonic resonance that occurs when the substrate is illuminated with an excitation wavelength is simulated with the FDTD method. The field intensity distributions inside the semicontinuous films are calculated by using SEM images of real samples, and we found good agreement between the experiments and the numerical results. 


\section{EXPERIMENTAL RESULTS OF OBLIQUELY DEPOSITED SERS SUBSTRATES}

Four SERS substrates that were fabricated by oblique angular deposition (OAD) and serial bideposition (SBD) were analyzed herein. OAD is a physical deposition technique in which the substrate is tilted at an angle with respect to the source flux. The shadowing effect for random nucleation granules with respect to the flux vapor causes nanorods to grow toward the incident deposition flux. ${ }^{16} \mathrm{SBD}$ is an alternative OAD method in which the incident deposition flux is supplied from two opposite directions in sequence (first from one direction, and then from the opposite direction) with respect to the substrate normal by rotating the substrate $180^{\circ}$ each step. ${ }^{17}$ In this case, the SBD was applied to form zig-zag structured templates.

One of the four samples (sample A) was a semicontinuous gold film that was obliquely deposited on a glass substrate. The other three samples were prepared by first growing a zig-zag $\mathrm{SiO}_{2}$ template layer and then obliquely depositing a thin $\mathrm{Au}$ film on top of the $\mathrm{SiO}_{2}$ columns. The zig-zag $\mathrm{SiO}_{2}$ template layers with various different thicknesses $(100,300$, and $500 \mathrm{~nm}$ for samples $\mathrm{B}, \mathrm{C}$, and $\mathrm{D}$, respectively) were grown using the SBD technique, and the zig-zag period was controlled to be less than $20 \mathrm{~nm}$. Then, Au was evaporated at a deposition angle $\left(\alpha_{\mathrm{Au}}\right)$ of $73.4^{\circ}$ with the same deposition plane as that of the $\mathrm{SiO}_{2}$ template layer. A small gold film was then attached to the fabricated template layer.

Figure 1 shows the morphology of a typical $\mathrm{SiO}_{2}$ template layer. The fan-out effect ${ }^{16}$ arises from the anisotropy of the shadowing effect, and it causes anisotropic broadening of the nano-columns. The columns develop a highly elliptical cross-section with the long axis in the direction perpendicular to the plane of the flux incidence defined by the directions of the deposited flux and the surface normal. The anisotropic broadening is proportional to the thickness. Serious fanning of the columns would cause the neighboring columns to connect together. As the thickness of the zig-zag template layer increases over $200 \mathrm{~nm}$, the adjacent columns connect to form a sheet structure as observed from the top view. The average width and length of the sheets vary with the film's thickness. Further deposited gold first forms discontinuous particles, and then forms a semicontinuous distribution that is elongated along the deposition plane of the $\mathrm{SiO}_{2}$. For a given amount of deposited gold such that the four samples all have the same mass thickness, the number and average width of these discontinuous gaps fall as the thickness of the template increases from 100 to $500 \mathrm{~nm}$. The filling factor here is defined as the proportion of the metal contained in the metaldielectric composite film. The actual thickness of the gold layer $d_{A, B, C, D}$ was estimated from the mass thickness $t_{\mathrm{Au}}$ and the filling factor $f_{A, B, C, D}$ to be $d_{A, B, C, D}=t_{\mathrm{Au}} / f_{A, B, C, D}$. From the filling factors of gold on the surfaces $\left(f_{A}=0.73\right.$, $f_{B}=0.52, f_{C}=0.48$, and $f_{D}=0.45$ for samples $\mathrm{A}, \mathrm{B}, \mathrm{C}$, and $\mathrm{D}$, respectively, as shown in Figs. 2(a)-2(h)), the actual thicknesses of the $\mathrm{Au}$ films were determined to be $d_{\mathrm{Au}, A}=t_{\mathrm{Au}} / f_{A}=13.7 \mathrm{~nm}, d_{\mathrm{Au}, B}=t_{\mathrm{Au}} / f_{B}=19.2 \mathrm{~nm}, \quad d_{\mathrm{Au}, C}$ $=t_{\mathrm{Au}} / f_{C}=20.8 \mathrm{~nm}, \quad$ and $\quad d_{\mathrm{Au}, D}=t_{\mathrm{Au}} / f_{D}=22.2 \mathrm{~nm} \quad$ as shown in Table I. In Sec. III, the field intensity distribution
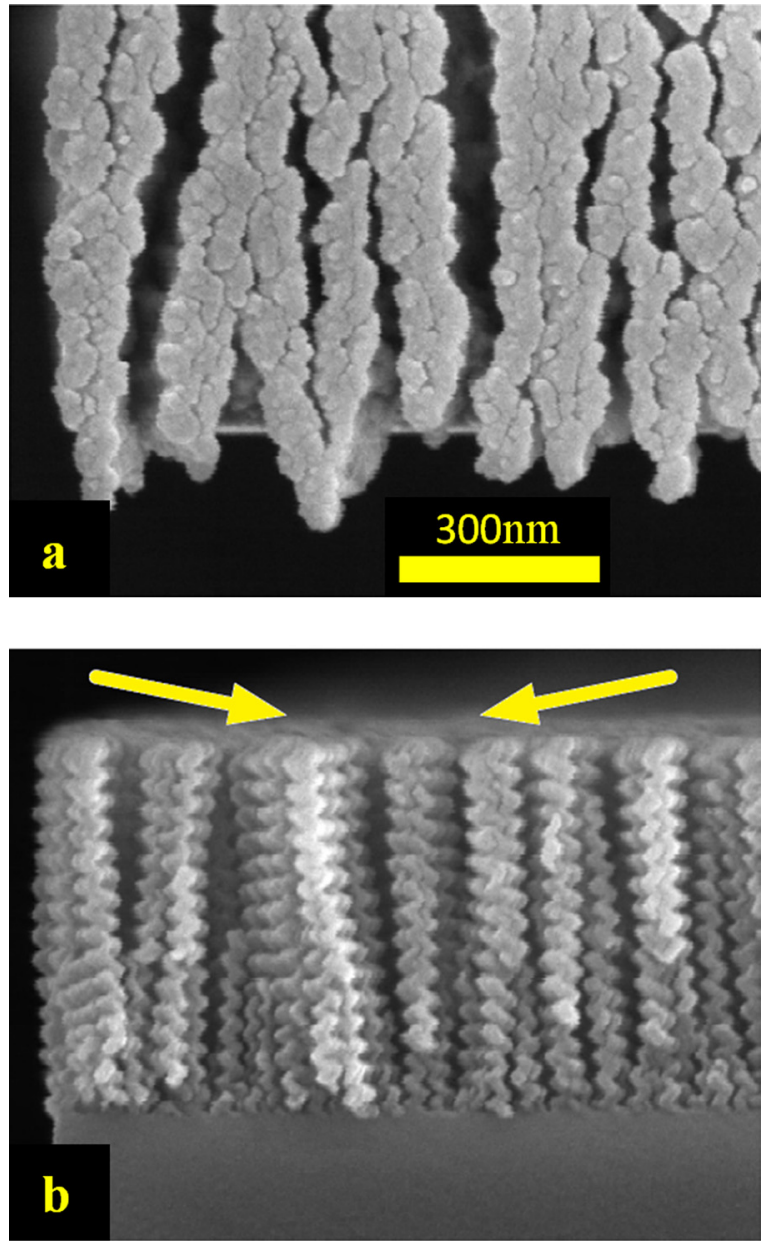

FIG. 1. SEM images of the $\mathrm{SiO}_{2}$ template layer: (a) the surface and (b) the cross-section fractured parallel to the deposition plane of the $\mathrm{SiO}_{2}$. The arrows indicate the deposition directions of the $\mathrm{SiO}_{2}$.

among the gold layer is calculated by dividing the layer into cubic cells, and the grid size for each cell is $2 \mathrm{~nm}$. Whether the material inside the cell is metal or air is chosen according to the morphology of SEM image.

The in situ measurements of SERS were carried out by immersing the Au nanorod arrays in a 4,4'-bipyridine (BiPy) water solution. Raman spectroscopy was performed using an excitation laser source with a wavelength of $785 \mathrm{~nm}$ and a power of $1.5 \mathrm{~mW}$. The $s$-polarization of the excitation light is defined as the polarization in which the electric field oscillates parallel to the deposition plane. The microscope has a $60 \times$ objective (the NA of which is 0.7 ) and a confocal pinhole with a diameter of $1.1 \mathrm{~mm}$. The SERS intensity achieved using $s$-polarized light has been demonstrated to be considerably higher than that achieved using $p$-polarized light. The results reveal that the intensity of Raman scattering is proportional to the thickness of the template. Fig. 2(i) presents the experimental results of the SERS measurement. ${ }^{15}$

\section{NEAR-FIELD SIMULATION}

\section{A. Modeling of obliquely deposited SERS substrates}

With respect to applications of SERS, local-field enhancements in the near-field region are of greater interest 


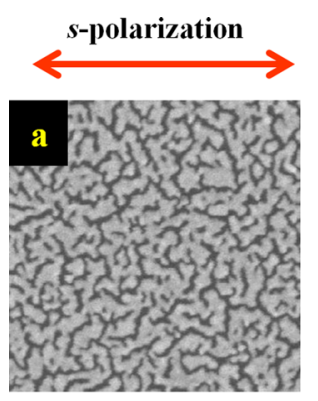

\section{$300 \mathrm{~nm}$}
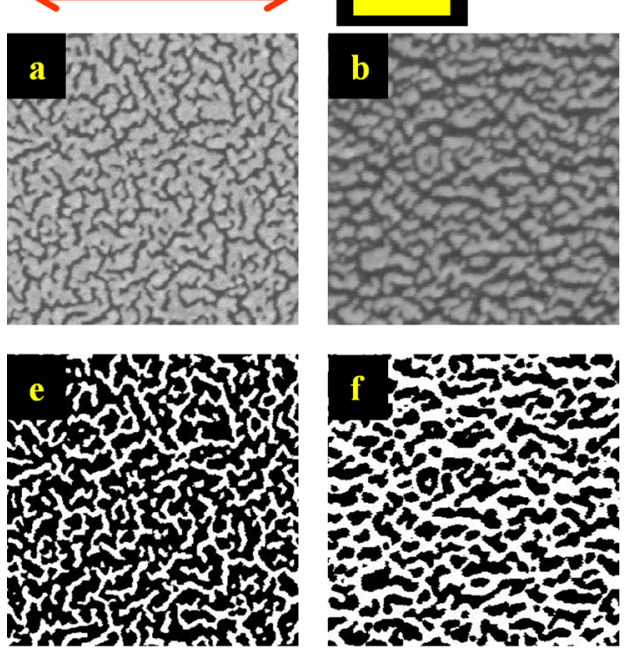
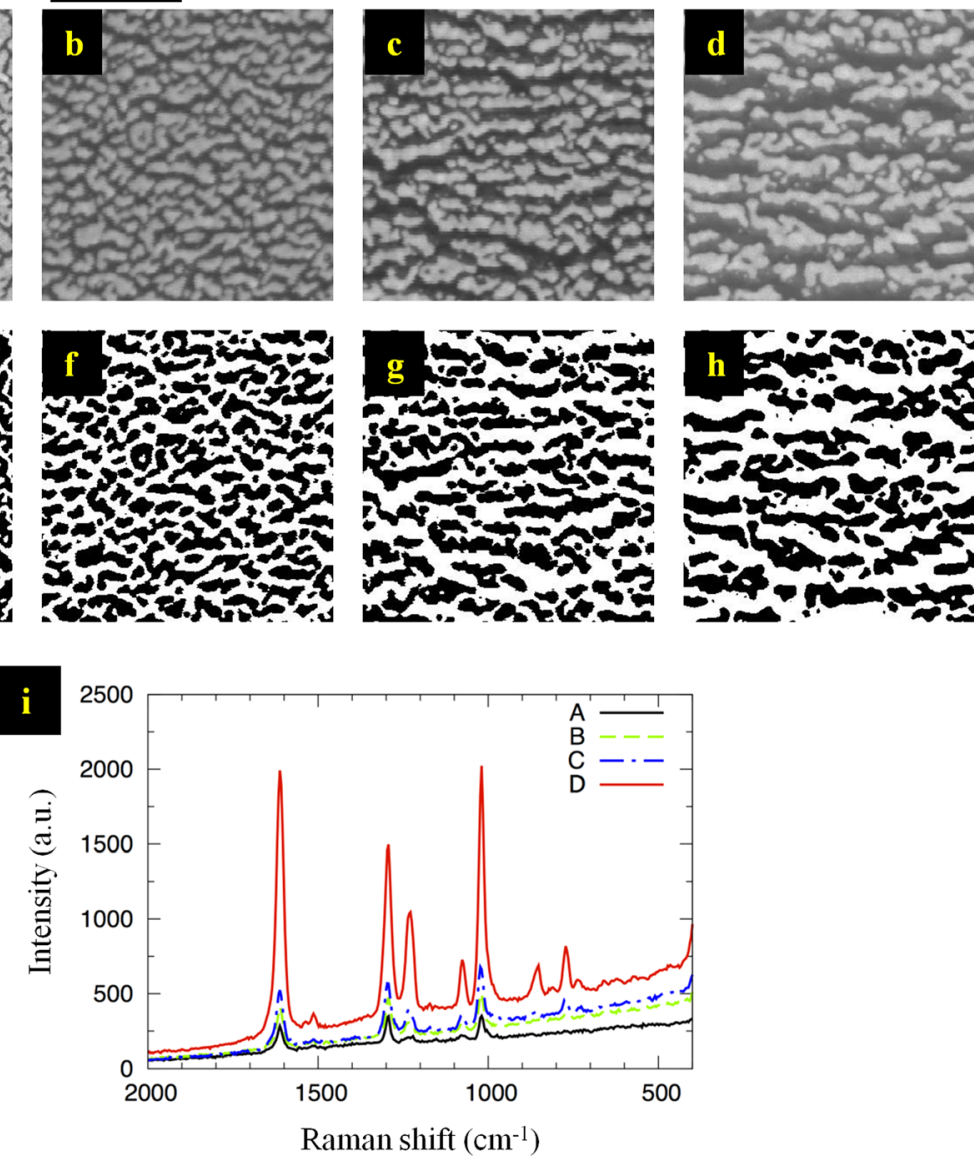

FIG. 2. (a) SEM and corresponding binarized SEM images of sample A ((a) and (e)), sample B ((b) and (f)), sample $\mathrm{C}((\mathrm{c})$ and $(\mathrm{g}))$, and sample D ((d) and (h)), respectively. In the binarized images, (e)-(h), the white regions represent air, while the black regions represent gold. The electric field of the $s$-polarized light is perpendicular to the deposition plane of the vapor flux. (i) Polarization-dependent Raman spectra of BiPy solution $\left(10^{-3} \mathrm{M}\right)$. than the far-field spectra. FDTD software, called FULLwAVE 6.0 and made by R-Soft, was utilized to determine the localized electric field based on the scanning electron microscopic (SEM) image. The top-view SEM images were converted to binary (black/white) images using an appropriate threshold to distinguish Au from air. Figs. 2(a)-2(h) show the original SEM images of the fabricated samples A, B, C, and D, which correspond to the binarized images in Figs. 2(e)-2(h), respectively. The structures for the simulation were approached by treating each sample as an upright columnar array with $\mathrm{Au}$ on the top. The cross-section of each column was determined from the top-view binary image and was invariant with altitude. Notably, the simulated area differed from the analyzed area. The simulation area was $900 \mathrm{~nm}$ $\times 900 \mathrm{~nm}$ and was delineated by the red boundary in Fig.3(a). Since a perfect matching boundary would affect the field distribution around the boundary, the analyzed area was shrunk to $700 \mathrm{~nm} \times 700 \mathrm{~nm}$ and delineated by the blue boundary in Fig. 3(a).

TABLE I. The filling factor and thickness of gold film for each sample.

\begin{tabular}{lcccc}
\hline \hline Sample ID & $\alpha_{\mathrm{Au}}$ & $t_{\mathrm{Au}}$ & $f$ & $d_{\mathrm{Au}}$ \\
\hline Sample A & $73.4^{\circ}$ & $10 \mathrm{~nm}$ & 0.73 & $13.7 \mathrm{~nm}$ \\
Sample B & $73.4^{\circ}$ & $10 \mathrm{~nm}$ & 0.52 & $19.2 \mathrm{~nm}$ \\
Sample C & $73.4^{\circ}$ & $10 \mathrm{~nm}$ & 0.48 & $20.8 \mathrm{~nm}$ \\
Sample D & $73.4^{\circ}$ & $10 \mathrm{~nm}$ & 0.45 & $22.2 \mathrm{~nm}$ \\
\hline \hline
\end{tabular}

The system coordinates $(x, y, z)$ were defined as shown in Figs. 3(a) and 3(b); the deposition plane was the $y$ - $z$ plane, and the $z$-axis was normal to the surface. The excitation light source was a laser with a wavelength of $785 \mathrm{~nm}$. The ray was normally incident with $s$-polarization along the $x$-axis. The Lorentz-Drude model $^{18}$ and Sellmeier equation ${ }^{19}$ were utilized to specify the dispersion relation of the dielectric constants of $\mathrm{Au}$ and $\mathrm{SiO}_{2}$, respectively. In the simulation, as the electromagnetic wave propagated through the $\mathrm{Au}$ film, the intensity of the oscillating electric field varied with time. Data were extracted when the oscillating fields reached a steady state. As the average electric field over the whole film varied steadily with time, the field distribution when the average field reached the maximum was analyzed. The intensities of all cells in the gold layer at the chosen time were calculated for each sample. The grid size for each cell was $2 \mathrm{~nm}$; therefore, each gold layer was divided into sub-layers to perform the simulation: there were $7,9,10$, and 11 sublayers in samples A, B, C, and D, respectively.

\section{B. Results and discussion of the near-field simulation}

\section{Near-field distribution}

The intensity is defined as $I=\left|E_{L}\right|^{2} /\left|E_{0}\right|^{2}$, where $E_{L}$ and $E_{0}$ are the local and incident fields, respectively. Figure 4 shows the intensity maps at the depth $z$ at which the intensity is maximum for each sample. The maximum value of the color axis is set to 1000 . The total area associated with 
(a)
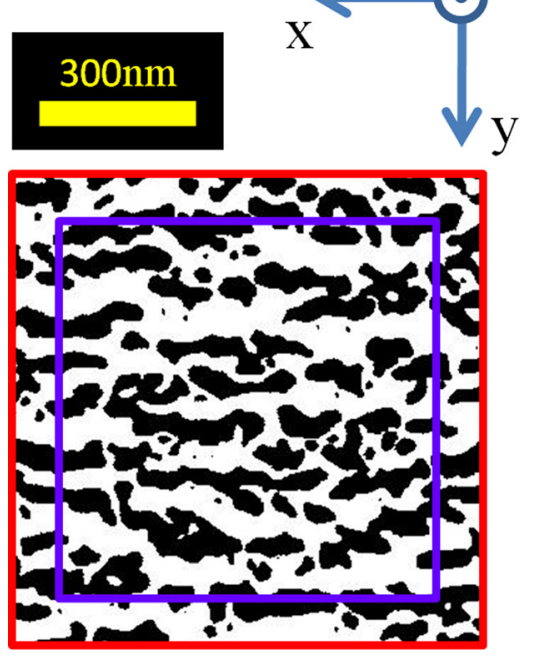

(b)

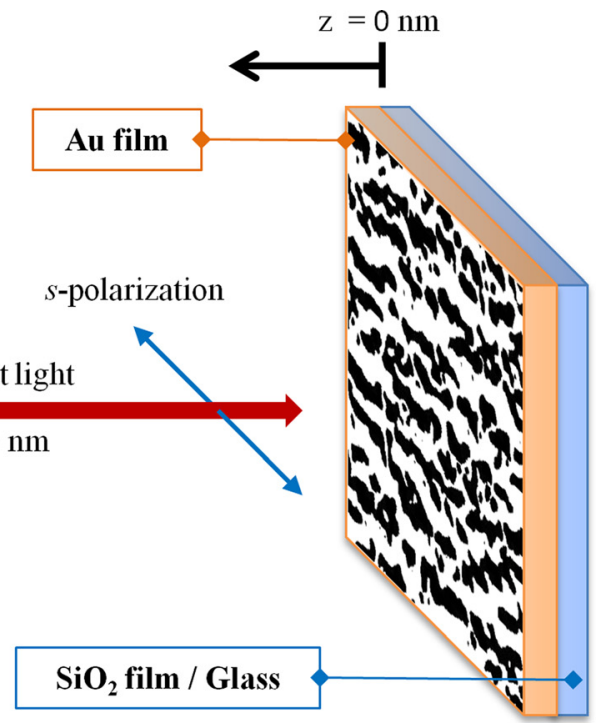

FIG. 3. (a) System coordinates $(x, y, z)$. (b) The direction of incident light was along the $z$-axis and the electric field of the $s$-polarized light was parallel to the $x$-axis. intensities over 1000 is around $3500 \mathrm{~nm}^{2}$ for each sample. Hot spots were present near the small gaps between the metal clusters, especially when the gaps were smaller than $2 \mathrm{~nm}$. Figures 5(a) and 5(b) plot the average field intensity $\left.I_{a v}^{j}(z)\right|_{j=A, B, C, D}$ and the maximum field intensity $\left.I_{\max }^{j}(z)\right|_{j=A, B, C, D}$ as functions of the depth $z$, respectively. For the four samples, the functions $I_{a v}(z)$ have similar trends: two peaks are obtained near the substrate and the air, as shown in Fig. 5(a). The magnitudes of the intensities of samples B, C, and D satisfy the relationship
$I_{a v}^{B}(z)>I_{a v}^{C}(z)>I_{a v}^{D}(z)$, and their values are all within the small ranges [32, 38], [31, 34], and [28, 32], respectively, between two peak values near both sides of the $\mathrm{Au}$ film. However, the $I_{\max }^{j}(z)$ values for samples B, C, and D are in the order $I_{\max }^{B}(z)<I_{\max }^{C}(z)<I_{\text {max }}^{D}(z)$, as shown in Fig. 5(b). The $I_{\max }(z)$ of sample $\mathrm{C}$ is very close to that of sample D from $z=0 \mathrm{~nm}$ to $z=14 \mathrm{~nm}$. The maximum values $I_{\max }^{B}(z)$, $I_{\max }^{C}(z)$, and $I_{\max }^{D}(z)$ are 13618 at $z=18 \mathrm{~nm}, 21852$ at $z=18 \mathrm{~nm}$, and 30430 at $20 \mathrm{~nm}$, respectively. The three maxima are found near the interface between metal and air.
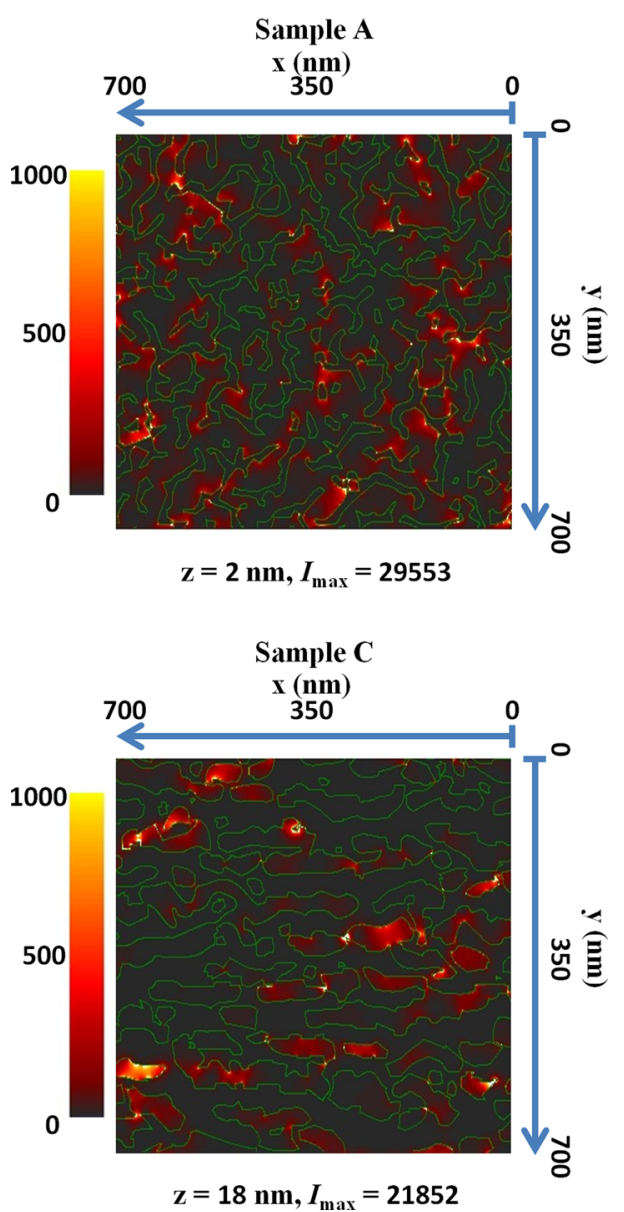
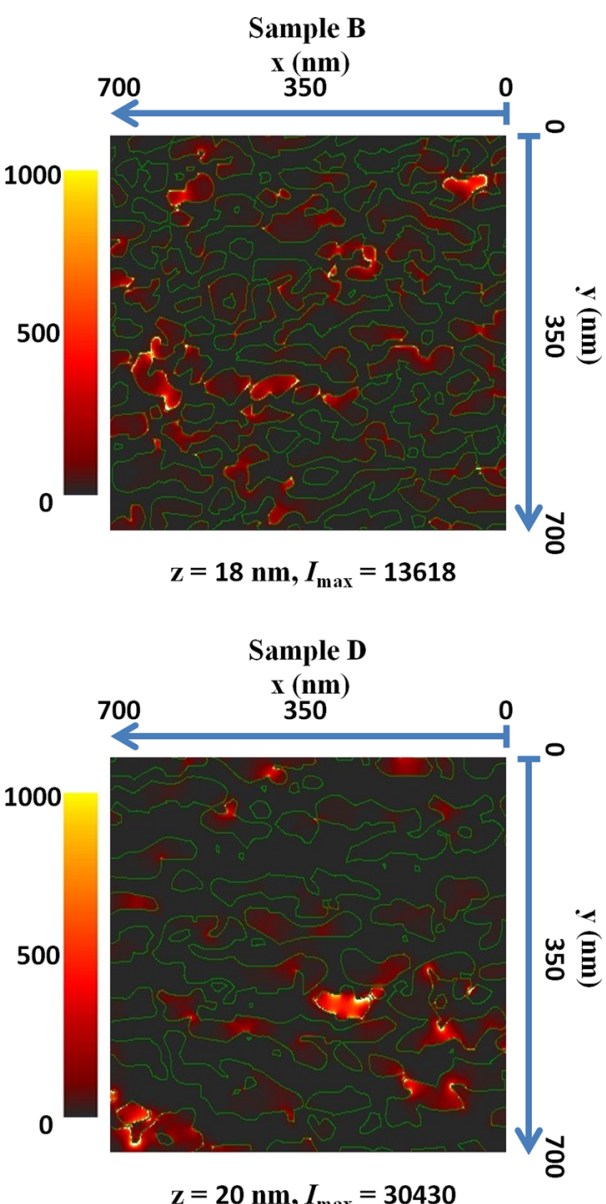

FIG. 4. Local intensity maps at the depth $z$ at which the intensity is maximum for samples A, B, C, and D. The green outlines are the boundaries of the gold clusters. 

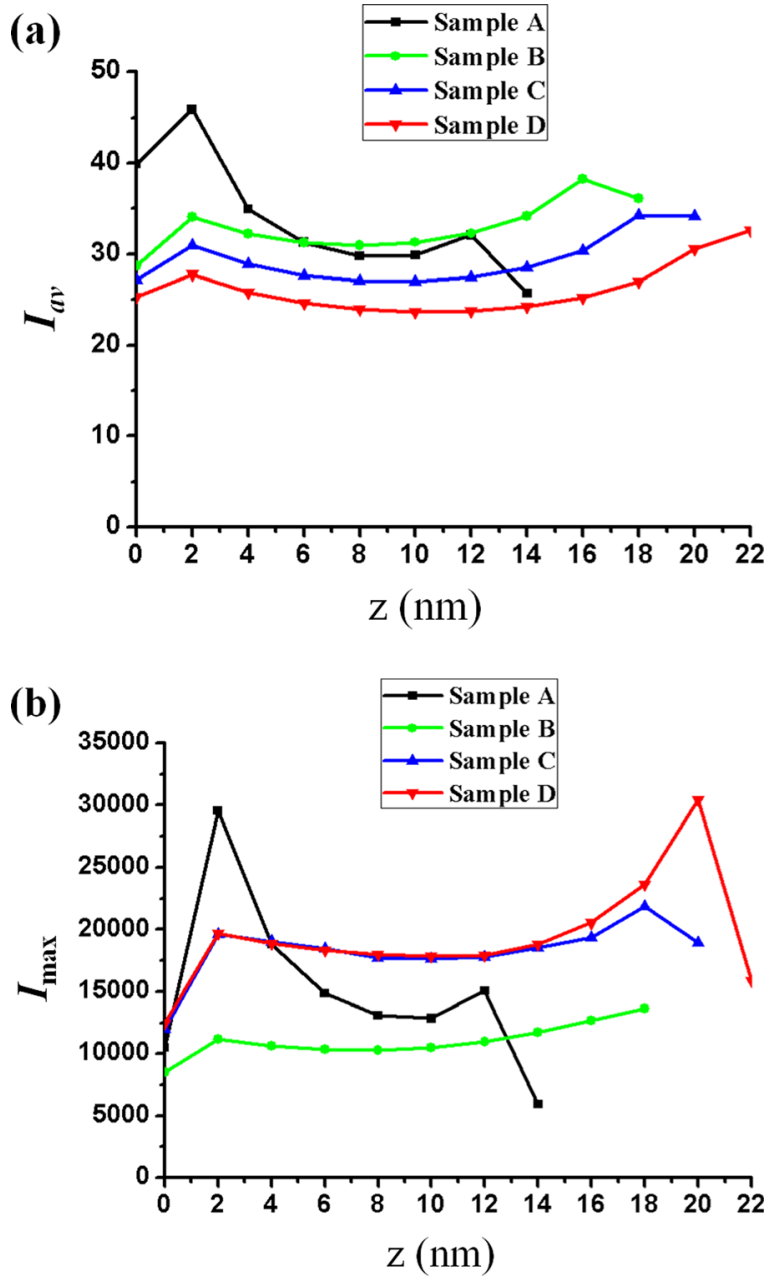

FIG. 5. (a) Average intensity $I_{a v}(z)$ and (b) maximum intensity $I_{\max }(z)$ are functions of $z$ for samples A, B, C, and D, respectively. Metal/substrate interface is at $z=0$.

Although the maximum intensity of sample A exceeds those of the other three samples, it is located at a distance of $2 \mathrm{~nm}$ from the surface of the substrate. The dissipation of the gold layer reduces the output scattered intensity of light from the hot spots close to the substrate.

\section{SERS enhancement factor}

The electromagnetic enhancement factor $(\mathrm{EF})$ has been reported to describe the influence of near-field enhancement on SERS. The EF in SERS has a dependence that is close to the fourth power of the localized field, $G \approx\left(\left|E_{L}\right|^{4} /\left|E_{0}\right|^{4}\right)$. The value of the intensity in each cell is recorded to calculate the maximum, the average, and the sum of the $G$ values over the gold layer. The maximum of $G, G_{\max }$; the average of $G$, $G_{a v}$; and the sum over the $G$ values, $G_{\text {sum }}$, were calculated based on the following definitions:

$$
\begin{gathered}
G_{\max }=\left|\frac{E_{L(\max )}}{E_{0}}\right|^{4}, \\
G_{\text {sum }}=\sum_{i=1}^{i=N}\left|\frac{E_{L}(i)}{E_{0}}\right|^{4},
\end{gathered}
$$

TABLE II. $G_{a v}, G_{\max }$, and $G_{s u m}$ for the gold layer for samples A, B, C, and $\mathrm{D}$, when s-polarized light with an excitation wavelength of $785 \mathrm{~nm}$ was used.

\begin{tabular}{lccc}
\hline \hline Sample ID & $G_{a v}$ & $G_{\max }$ & $G_{\text {sum }}$ \\
\hline Sample A & $1.81 \times 10^{4}$ & $8.73 \times 10^{8}$ & $1.78 \times 10^{10}$ \\
Sample B & $1.64 \times 10^{4}$ & $1.85 \times 10^{8}$ & $2.02 \times 10^{10}$ \\
Sample C & $2.72 \times 10^{4}$ & $4.78 \times 10^{8}$ & $3.69 \times 10^{10}$ \\
Sample D & $2.81 \times 10^{4}$ & $9.26 \times 10^{8}$ & $4.15 \times 10^{10}$ \\
\hline \hline & \\
& $G_{a v}=\frac{1}{N} \sum_{i=1}^{i=N}\left|\frac{E_{L}(i)}{E_{0}}\right|^{4}$,
\end{tabular}

where $N$ is the total number of cells in the gold layer.

Table II presents $G_{a v}, G_{\max }$, and $G_{\text {sum }}$. The maximum values of $G$ and $G_{\max }$ of the four samples are in the range from $1.85 \times 10^{8}$ for sample B to $9.26 \times 10^{8}$ for sample D. $G_{\text {sum }}^{j}$ is derived by summing $G$ over all cells for sample $j$ in the gold layer. The term $G_{a v}$ denotes the average of $G$ over all cells and can be derived by dividing $G_{\text {sum }}$ by the total number of cells, $N$. The value of $G_{a v}$, ranging from $1.64 \times 10^{4}$ for sample B to $2.81 \times 10^{4}$ for sample $\mathrm{D}$, is four orders of magnitude less than $G_{\max }$. The fact that sample D has the highest values of $G_{\max }, G_{a v}$, and $G_{\text {sum }}$ among the four samples is consistent with the experimental determination that sample D had the strongest Raman signal. The magnitudes of $G_{\text {sum }}$ follow the order $G_{\text {sum }}^{D}>G_{\text {sum }}^{C}>G_{\text {sum }}^{B}>G_{\text {sum }}^{A}$, which matches the measured ordering of strengths of the Raman signals.

A comparison with experimental results indicates that the electric field intensity obtained through the FDTD simulation, $I_{\max }(z)$, explains well the ordering of SERS signals from samples B, C, and D. Calculating the enhancement factor $G$ reveals that the ordering of $G_{\text {sum }}$ among the four samples is the same as that of the measured SERS. Since the factor $G$ scales as the fourth power of the electric field, it magnifies the effect of those fields with large magnitudes. The $G_{a v}$ of the four samples follows the order $G_{a v}^{B}<G_{a v}^{A}<G_{a v}^{C}<G_{a v}^{D}$, because sample $B$ is thicker than sample $A$, and so has more hot spots, explaining the experimental result.

\section{CONCLUSION}

In conclusion, FDTD was utilized to simulate the distribution of the electric field in an obliquely deposited SERS substrate under illumination by excitation light. The field intensity remains around a particular value within the gold layer supported by a $\mathrm{SiO}_{2}$ template. Unlike the single gold layer on the substrate, in which the field intensity decays from the substrate to the air, two peaks of field intensity are obtained near both sides of the gold layer on a $\mathrm{SiO}_{2}$ template. The simulation indicates that an enhancement of the field intensity within a small area near the metal surface will cause a strong SERS signal. Therefore, the distribution of hot spots has a greater influence on SERS than the average intensity value. The calculated $G$ factors $G_{\text {sum }}$ of the four samples give the same ordering as that of the SERS signals because the fourth power of the electric field will enhance the contribution of the maximum fields at each hot spot. This analysis 
is helpful in understanding the mechanism by which SERS is produced from a nanostructure. This approach can be useful in comparing SERS measurements between different SERS substrates.

${ }^{1}$ F. Yan, M. B. Wabuyele, G. D. Griffin, A. A. Vass, and T. Vo-Dinh, IEEE Sens. J. 5, 665 (2005)

${ }^{2}$ T. Vo-Dinh, F. Yan, and M. B. Wabuyele, J. Raman Spectrosc. 36, 640 (2005).

${ }^{3}$ K. K. Hering, R. Möller, W. Fritzsche, and J. Popp, ChemPhysChem 9, 867 (2008).

${ }^{4}$ J. Guicheteau, L. Argue, D. Emge, A. Hyre, M. Jacobson, and S. Christesen, Appl. Spectrosc. 62, 267 (2008).

${ }^{5}$ L. Qin, M. J. Banholzer, J. E. Millstone, and C. A. Mirkin, Nano Lett. 7, 3849 (2007).

${ }^{6}$ H. Zhang, M. H. Harpster, H. J. Park, P. A. Johnson, and W. C. Wilson, Anal. Chem. 83, 254 (2011).

${ }^{7}$ R. A. Ando, N. P. W. Pieczonka, P. S. Santos, and R. F. Aroca, Phys. Chem. Chem. Phys. 11, 7505 (2009).
${ }^{8}$ C. J. Orendorff, L. Gearheart, N. R. Jana, and C. J. Murphy, Phys. Chem. Chem. Phys. 8, 165 (2006).

${ }^{9}$ G. Laurent, N. Felidj, J. Aubard, G. Levi, J. R. Krenn, A. Hohenau, G. Schider, A. Leitner, and F. R. Aussenegg, Phys. Rev. B 71, 45430 (2005).

${ }^{10}$ Q. Yu, S. Braswell, B. Christin, J. Xu, P. M. Wallace, H. Gong, and D. Kaminsky, Nanotechnology 21, 355301 (2010).

${ }^{11}$ V. P. Drachev, M. Thoreson, E. Khaliullin, A. K. Sarychev, D. Zhang, D. Ben-Amotz, and V. M. Shalaev, Proc. SPIE 5221, 76 (2003).

${ }^{12}$ J. L. Martínes, Y. Gao, and T. López-Ríos, Phys. Rev. B 33, 5917 (1986).

${ }^{13}$ S. B. Chaney, Z. Y. Zhang, and Y. P. Zhao, Appl. Phys. Lett. 89, 53117 (2006).

${ }^{14}$ M. Suzuki, W. Maekita, Y. Wada, K. Nakajima, K. Kimura, T. Fukuoka, and Y. Mori, Appl. Phys. Lett. 88, 203121 (2006).

${ }^{15}$ M. Suzuki, K. Nakajima, K. Kimura, T. Fukuoka, and Y. Mori, Anal. Sci. 23, 829 (2007).

${ }^{16}$ D.-X. Ye1, T. Karabacak, R. C. Picu, G.-C. Wang, and T.-M. Lu, Nanotechnology 16, 1717 (2005)

${ }^{17}$ I. Hodgkinson and Q. H. Wu, Appl. Opt. 38, 3621 (1999).

${ }^{18}$ A. D. Rakic, A. B. Djurisic, J. M. Elazar, and M. L. Majewski, Appl. Opt. 37, 5271 (1998).

${ }^{19}$ I. H. Malitson, J. Opt. Soc. Am. 55, 10 (1965). 